\title{
Lidil
}

Revue de linguistique et de didactique des langues

$45 \mid 2012$

Pratiques de formation à la lecture-écriture des adultes en parcours d'insertion

\section{Adaptation des formateurs en milieu professionnel dans un environnement en mutation}

\section{Christophe Portefin}

\section{CpenEdition}

\section{Journals}

Édition électronique

URL : http://journals.openedition.org/lidil/3176

DOI : 10.4000/lidil.3176

ISSN : 1960-6052

Éditeur

UGA Éditions/Université Grenoble Alpes

Édition imprimée

Date de publication : 15 mai 2012

Pagination : $27-49$

ISBN : 978-2-84310-226-4

ISSN : $1146-6480$

Référence électronique

Christophe Portefin, «Adaptation des formateurs en milieu professionnel dans un environnement en mutation », Lidil [En ligne], 45 | 2012, mis en ligne le 15 novembre 2013, consulté le 01 mai 2019. URL http://journals.openedition.org/lidil/3176 ; DOI : 10.4000/lidil.3176

(C) Lidil 


\title{
Adaptation des formateurs en milieu professionnel dans un environnement en mutation
}

\author{
Christophe Portefin*
}

\begin{abstract}
RÉSUMÉ
Depuis quelques années, les dispositifs de formation professionnelle continue sont en pleine mutation. Les formateurs intervenant en milieu professionnel dans l'apprentissage de la lecture-écriture auprès des adultes se retrouvent au centre d'un système complexe mouvant au sein duquel ils doivent faire évoluer leurs pratiques. Après avoir montré les orientations actuelles du métier, l'enquête rend compte, au travers d'une analyse qualitative réalisée auprès de formateurs, de leur adaptation à ces évolutions.
\end{abstract}

\begin{abstract}
Over the past years, the organizations of vocational training have seen some radical transformations. Trainers who teach reading and writing to adult workers face an ongoing complex system which forces them to adapt their. The survey first highlights the current trends of the job and then a qualitative analysis run on the trainers shows how they adapt to the changes.
\end{abstract}

Apprendre à des adultes à lire et écrire dans le cadre de leur travail demande aux formateurs/formatrices ${ }^{1}$ de se situer au centre de dispositifs de formation inscrits dans un environnement socioprofessionnel,

* Doctorant en «Didactique des langues et des cultures » en codirection (équipes de recherche DILTEC (Didactique des langues, des textes et des cultures), Paris 3 ; PLIDAM (Pluralité des Langues et des Identités : Didactique, Acquisition, Médiations), INALCO). Coordinateur pédagogique/ formateur au sein d'ACCENTONIC.

1. Pour plus de facilité, nous utiliserons désormais le masculin formateur( $(s)$, indifférencié dans une profession (pourtant) majoritairement féminine. 
aujourd'hui en pleine mutation. Non seulement les formateurs sont passés d'une didactique du français général à celle d'un français professionnel depuis la loi du 4 mai 2004 relative à la formation professionnelle $^{2}$, loi dans laquelle le français est devenu une compétence professionnelle au même titre que les compétences plus techniques, mais, de plus, ils doivent désormais passer d'un statut de formateur de langue à celui de formateur aux compétences clés / savoirs de base / savoirs fondamentaux en contexte professionnel (domaine de formation englobant entre autres le français / les mathématiques / la bureautique / le cadre réglementaire et destiné principalement aux personnes de bas niveau de qualification). Ceci demande adaptation, utilisation de nouveaux outils et référentiels, développement d'autres compétences, un véritable changement en profondeur du métier. Comment la conception de la formation linguistique et les pratiques afférentes sont-elles affectées par cette mutation en cours du contexte? Comment les formateurs transforment-ils ou non leurs pratiques? Ce texte se propose dans un premier temps de rendre compte des aspects institutionnels de cette mutation. Dans un deuxième temps il présentera les choix méthodologiques d'une recherche de terrain visant à analyser l'adaptation de formateurs intervenant dans des dispositifs de formation pour quatre branches professionnelles distinctes. La troisième partie sera consacrée à la présentation d'une partie des résultats de cette enquête.

\section{Un environnement complexe}

Former des salariés en contexte professionnel participe au dispositif général de la formation professionnelle continue (désormais FPC). À partir du moment où un formateur intervient avec des stagiaires dans le cadre d'une action de formation, cela signifie que tout un processus en amont a rendu possible la mise en place de cette formation. Sans revenir en arrière sur l'histoire de la FPC, il convient de retenir que, depuis la loi du 16 juillet 1971, les entreprises ont l'obligation de cotiser chaque mois pour la formation de leurs salariés. Soit les entreprises prévoient

2. Loi n ${ }^{\circ}$ 2004-391 relative à la formation professionnelle tout au long de la vie et au dialogue social. Ce droit a été inscrit dans le Code du Travail - article L6111-2: «Les actions de lutte contre l'illettrisme et en faveur de l'apprentissage de la langue française font partie de la formation professionnelle tout au long de la vie.» 
directement ce budget «plan de formation annuel » dans leurs comptes, soit le plus souvent elles deviennent adhérentes d'organismes paritaires collecteurs agréés (désormais OPCA), sortes de banques de la FPC chargées d'encaisser les fonds et de proposer en contrepartie des actions de formation à leurs adhérents. Ces OPCA sont rattachés directement à des branches professionnelles comme le BTP, la métallurgie, les artistes ou bien l'hôtellerie-restauration. Pour qu'une prestation de formation se réalise, il arrive que les OPCA labellisent directement des organismes de formation (désormais OF) et/ou rédigent des appels d'offre (désormais AO) puis sélectionnent les OF gagnants.

Les formateurs peuvent être, eux aussi, habilités à réaliser les formations, notamment lors de sessions de formations de formateurs réalisées par des prestataires de service pour les OPCA, des cabinets de consultants spécialisés dans des formations compétences clés / savoirs de base. Comme on peut le voir, ce type de formation mobilise beaucoup d'acteurs différents, le formateur et les stagiaires se retrouvant au milieu d'un environnement très complexe au sein duquel tous interagissent.

\section{Un environnement en mutation}

À la complexité de cet environnement, il faut ajouter son évolution normale au fil des ans et surtout de grands changements au cours de la dernière décennie. Depuis le début des années 2000, l'ouverture des marchés publics à la concurrence et aux $\mathrm{AO}$ a profondément bouleversé l'habitus et le fonctionnement des OF. Le marché est devenu concurrentiel et les attentes de pouvoirs publics et des OPCA ont fait changer la façon de travailler des OF et, de facto, des formateurs. On a alors parlé d'une nécessaire «professionnalisation du métier du formateur en formation de base, des individus, mais aussi de professionnalisation des structures » (Maroun, 2006 : 74), d'où la mise en place d'un Forum permanent des pratiques dans lequel V. Leclerc animait l'atelier sur la professionnalisation des intervenants au sein de l'Agence nationale de Lutte contre l'Illettrisme (désormais ANLCI) dès 2004, année de promulgation de la loi relative à la formation professionnelle ${ }^{3}$. Alors qu'auparavant les marchés étaient plus ou moins acquis, désormais les OF doivent se battre et ne peuvent plus se contenter des marchés publics, ce qui a entrainé la disparition de nombreux OF. Si certains ont résisté et remporté des marchés, nous pouvons émettre l'hypothèse qu'ils le

3. Voir introduction à ce même article. 
doivent à des changements de pratiques, notamment en amont de la formation. En cela nous partageons l'avis de Boutet (2006 : 38) qui déclare qu'

avant toute démarche de formation ou de remédiation, d'ingénierie de formation, il [lui] semble qu'il faut passer par l'observation, la description et l'analyse du travail réel et en l'occurrence du travail en tant que travail langagier.

Des formateurs ont ainsi été amenés à proposer de nouvelles actions de formation après analyse de besoins réels, en y développant plus formellement qu'auparavant les objectifs de formation, les méthodologies utilisées et les modalités d'évaluation afin de travailler en formation sur ce que Boutet (2006 : 39) appelle le « capital de communication ». De plus, depuis 2006, la mise en place du diplôme initial de langue française (DILF) puis du contrat d'accueil et d'intégration (CAI) dans le cadre d'une politique générale de maitrise de l'immigration, font que la langue française devient le sésame obligatoire des nouveaux migrants souhaitant rester en France. L'arrivée d'un nouveau gouvernement a confirmé cette orientation en renforçant le lien entre langue-travail-immigration professionnelle et intégration professionnelle au travers d'une

politique d'accueil et d'intégration [qui] doit reposer sur trois piliers essentiels : le partage de la langue française et des valeurs de la République, le travail et le logement [...]. Vous veillerez à ce que chaque migrant bénéficie d'un suivi individualisé de son parcours d'intégration : délivrance du visa de long séjour sous réserve de l'apprentissage du français et de la connaissance des valeurs de la République ${ }^{4} .$.

Confirmation étendue également à l'échelle européenne dans le Pacte européen sur l'immigration et l'asile de 2008 dans lequel « l'apprentissage de la langue du pays d'accueil et l'accès à l'emploi sont considérés comme des facteurs essentiels d'intégration ${ }^{5} »$.

Tout ceci a transformé également les OF, les portant sur des marchés sur lesquels les formateurs de FLE réalisent des formations plus ou moins longues de français pour l'obtention du DILF. Parallèlement à ces changements et en adéquation avec cette évolution, est apparu un nouveau champ en didactique des langues, la formation linguistique en

4. Lettre de mission du Premier ministre au ministre de l'Immigration, de l'Intégration, de l'Identité nationale et du Développement solidaire, 2009.

5. Souligné dans le texte de la plaquette officielle du ministère. 
contexte d'insertion, le français langue d'insertion (ou FLI ${ }^{6}$ ) qui « soustend une conception de la formation comme visant la dynamique de parcours de "mobilités sociolinguistiques", pour l'accomplissement desquels l'appropriation linguistique constitue une ressource sociale symbolique fondamentale » (Bretegnier, Etienne, Adami, 2011 : 14). Enfin, pendant cette même période, la FPC subit réformes sur réforme, fait l'objet de différents rapports (parlementaires, Cour des comptes, Conseil d'orientation pour l'emploi...), suivis par des lois qui recomposent le paysage de la FPC. Nous nous arrêterons sur la plus importante dans le champ qui nous occupe, l'Accord national interprofessionnel sur le développement de la formation tout au long de la vie professionnelle, la professionnalisation et la sécurisation des parcours professionnels (désormais ANI) de 20097 . Cet ANI a mis en relief la nécessité de définir au sein du monde professionnel un « socle commun de connaissances et de compétences ${ }^{8} »$ préconisant aux branches/OPCA le soin de s'en charger. Pour ce faire, a été créé le Fonds paritaire de sécurisation des parcours professionnels (désormais FPSPP) chargé, entre autres, de publier des $\mathrm{AO}$ relatifs à ces socles communs et donc d'allouer des fonds aux OPCA gagnants, le nombre d'OPCA diminuant pendant cette même période pour des raisons légales. Enfin, la législation liée à cet ANI a changé les modalités de collecte des fonds de formation faisant en sorte que les OPCA participent à la fois aux formations des salariés et des chômeurs, ce qui amène de nouveaux partenariats entre branches professionnelles et Pôle Emploi.

En conséquence, les OF qui pouvaient être habilités par certains OPCA peuvent se voir désormais privés de nouveaux marchés, les fonds de formation ayant diminué au profit des demandeurs d'emploi. Dans le même mouvement, les OPCA porteurs d'actions comme les savoirs de base ont été rachetés/absorbés par de plus gros OPCA (par exemple, l'OPCALIA a intégré le FAF Propreté, devenant ainsi OPCALIA Propreté) ou se sont regroupés (CONSTRUCTYS, OPCA du BTP comprend désormais FAF SAB, OPCA Bâtiment, OPCA Travaux Publics,

6. Nous tenons à préciser que nous situons cet acronyme conformément à l'ouvrage paru sous la direction de Bretegnier : Formation linguistique en contexte d'insertion. Compétences, posture, professionnalité : concevoir un cadre de référence (s), Berne, Peter Lang, 2011.

7. Loi 2009-1437 du 24 novembre 2009 en application depuis le $1^{\text {er janvier } 2010 .}$

8. Un socle pour la FPC différent de celui de l'Éducation nationale réservé à la formation initiale. 
GFC-BTP et AREF-BTP) ${ }^{9}$. Les formateurs doivent donc composer aujourd'hui dans cet environnement complexe en pleine mutation et nous partageons l'apport didactique du FLI dans le sens où il « doit être pensé comme interdisciplinaire, à la croisée de diverses approches qui permettent de l'appréhender et de la comprendre dans sa complexité » (Bretegnier, Etienne, Adami, 2011 : 14), notamment dans sa prise en compte des différents publics rencontrés dans les formations impulsées par les branches professionnelles :

migrants $[\ldots]$ contraints d'apprendre le français $[\ldots]$, personnes qui relèvent de l'illettrisme, [des publics qui] tous ont en commun d'être inscrits ou assimilés à des groupes sociaux et/ou socioprofessionnels qui les placent dans les rangs des catégories les plus populaires. (Ibid. : 22-23)

\section{Choix méthodologiques}

\section{Le terrain de notre recherche}

Afin de rendre compte de l'adaptation des formateurs, nous menons une recherche dans le cadre d'une thèse en cours en codirection au sein des laboratoires DILTEC et PLIDAM. Étant nous-même acteur de terrain en tant que responsable pédagogique / formateur d'actions de formation de français professionnel / compétences clés au sein d'un OF situé en Îlede-France, nous avons pu réaliser cette recherche non seulement au sein de cet $\mathrm{OF}$ mais aussi avec d'autres $\mathrm{OF}^{10}$ intervenant dans des actions de formation pour quatre branches professionnelles très éloignées les unes des autres : Propreté et Services Associés (OPCALIA Propreté), Industries Agroalimentaires (OPCALIM), Bâtiment et Travaux Publics (CONSTRUCTYS), Textile/Habillement/Cuir et secteurs connexes (OPCALIA FORTHAC) ${ }^{11}$.

9. OPCALIA est un OPCA interprofessionnel/interbranches, FAF pour Fonds d'assurance formation, SAB pour salariés de l'artisanat du BTP (bâtiment et des travaux publics), GFC pour Groupement pour la formation continue dans les industries du BTP, AREF pour Association régionale paritaire pour la formation continue.

10. Cette recherche a été facilitée grâce à l'existence de réseaux d'OF habilités par ces branches professionnelles.

11. OPCALIM pour Organisme paritaire collecteur agréé des industries alimentaires, de la coopération agricole et de l'alimentation en détail, FORTHAC pour Formation textile, habillement cuir et secteurs connexes. 


\begin{tabular}{|l|l|}
\hline OPCA & Dispositifs de formation \\
\hline $\begin{array}{l}\text { OPCALIA } \\
\text { PROPRETÉ }\end{array}$ & $\begin{array}{l}\text { MCCP (Maitrise des compétences clés de la } \\
\text { Propreté) }\end{array}$ \\
\hline OPCALIM & $\begin{array}{l}\text { MSF (Maitrise des savoirs fondamentaux) } \\
\text { MSF ALICIA (plateforme multimédia) }\end{array}$ \\
\hline CONSTRUCTYS & Formations TOP \\
\hline $\begin{array}{l}\text { OPCALIA } \\
\text { FORTHAC }\end{array}$ & $\begin{array}{l}\text { Démarche compétences clés en situation } \\
\text { professionnelle }\end{array}$ \\
\hline
\end{tabular}

Tableau 1. - Les formations concernées par l'enquête

Comme nous pouvons le voir dans le tableau 1, nous ne parlons plus ici de remises à niveau, ces dernières n'étant plus

de mise parce que, d'une part, le « niveau » augmentant sans cesse, il serait illusoire de penser le rattraper et, d'autre part, parce qu'il semble désormais inutile, et de toutes façons théoriquement et pratiquement très difficile, de définir un niveau seuil général pour les compétences en matière de maitrise de l'écrit. (Adami, 2010 : 7)

Nous ne parlons pas non plus d'alphabétisation et partageons le concept de littéracisation d'Adami (2009) qui peut concerner une partie du public bénéficiant de ce type de formations. Nous nous situons donc dans le champ du FLI en contexte professionnel, avec un ancrage évident dans le français langue professionnelle (désormais FLP) qui « vise la maitrise de la langue en situation de travail, tout en faisant l'hypothèse que les pratiques de travail se voient améliorées par le perfectionnement linguistique, du moment que ce perfectionnement respecte les logiques professionnelles » (Mourlon-Dallies, 2008 : 90). Cependant, en plus de la langue, les savoirs fondamentaux et les compétences clés concernent d'autres compétences à travailler, dispenser en formation comme la numératie ${ }^{12}$ ou l'informatique par exemple. Il apparait donc que notre

12. Le concept de numératie/numéracie n'est pas, selon nous, encore réellement stabilisé. Nous nous rapprochons dans sa définition de celle proposée par Ressources humaines et Développement des compétences Canada : « La numératie correspond à la capacité d'une personne de comprendre et d'utiliser des données mathématiques à l'école, au travail et dans la vie de tous les jours; par exemple, pour utiliser de la monnaie et établir des budgets, pour utiliser des mesures en cuisine ou pour lire une carte. » Disponible en ligne sur <http://www4.hrsdc.gc.ca/gl.4ss.1ry@-fra.jsp?wrd=Num\%E9ratie+ des+adultes\&iid=79> [consulté le 25 mars 2012]. 
terrain de recherche étire la didactique des langues à d'autres champs probables même si le français restera le seul lien véhiculaire entre toutes ces compétences clés par exemple. Nous pouvons donc supposer que l'adaptation des formateurs nécessitera probablement qu'eux-mêmes soient formés pour développer ces nouvelles actions de formation.

\section{La démarche}

Notre recherche s'inscrit dans une approche qualitative; nous nous inscrivons dans la continuité des travaux de l'ethnographie de la communication de Gumperz (1964) et Hymes (1974) pour lesquels l'ethnographie et la communication fournissent un cadre de référence plaçant la parole comme partie intégrante de la culture. Notre enquête porte sur un groupe connu de l'intérieur ce qui « est même un excellent moyen de pénétrer ces univers que de se soumettre au cycle des contingences qui marquent l'existence quotidienne de ceux qui y vivent » (Goffman, $1961: 37$ ). Notre observation est donc participante, notre but étant de « saisir le point de vue de l'indigène [ici les formateurs], ses rapports à la vie, de comprendre la vision de son monde » (Malinovski, 1963 : 81-82). De plus, notre ancrage professionnel en tant qu'acteur de terrain s'inscrit dans une "relation avec les acteurs [qui] est celle de quelqu'un qui se trouve dans le groupe avec la fonction d'observer, notre rôle est reconnu par les acteurs et est intégré dans le champ même de l'observation » comme l'entend Cambra Giné (2003 : 17) dans sa définition de l'approche ethnographique. Par ce fait, en tant qu'observateur participant, nous sommes aussi « une partie de son observation, et seule sa capacité à observer sa position dans la communauté indigène garantit l'objectivité de l'approche ethnographique » (Géraud, Leservoisier, Pottier, 1998 : 31). Soulignons par ailleurs que nous n'avons pas cherché à approcher un groupe inconnu puisque nous faisions déjà partie intégrante de ce groupe. C'est pourquoi nous nous intéresserons plus particulièrement aux formateurs intervenant auprès de salariés des branches citées ci-dessus.

\section{Les outils de recueil}

Afin de mesurer les changements dans leurs pratiques et leur adaptation, nous avons recueilli des données de nature différente ${ }^{13}$ :

- un corpus de traces écrites relatives à l'activité professionnelle sur le terrain sous la forme d'un journal de terrain « tenu très réguliè-

13. L'ensemble de ces données étant important, nous ferons figurer en annexe un extrait représentant les différents types de données recueillies. 
rement $[\ldots]$ avec une discipline devenue aussi naturelle que celle d'un drogué de jogging » (Winkin, 2001 : 146). Ce journal s'inscrit dans notre démarche ethnographique et nous partageons les fonctions proposées par Winkin (2001 : 147) du journal : « cathartique [...] le journal sera le lieu du corps-à-corps avec vous-même, face au monde social étudié / empirique. Vous y noterez tout ce qui va capter votre attention lors des séances d'observation / réflexive et analytique ». Ce travail d'écriture nous « permet à la fois de revivre une expérience et de l'objectiver [...] stratégie de décalage [...] d'autant plus nécessaire si l'on travaille au sein de sa propre société »(Winkin, 2001 : 163). Dans ce journal ont ainsi été notés des réflexions, remarques, propos de formateurs et d'autres intervenants évoluant dans cet environnement, notes écrites et recueillies lors de réunions pédagogiques, d'actions de formations de formateurs, de comités de suivi de formations, de 2008 à 2011;

- un corpus d'enregistrements sonores et vidéos : ces enregistrements ont été réalisés lors de séances de formation de façon formelle et lors des réunions précédentes en complément des traces écrites;

- des questionnaires distribués aux formateurs des OF concernés par notre enquête : à ce titre, nous avons pu recueillir six questionnaires sur les trente distribués au sein de quatre OF. Ces questionnaires se sont vus imposés suite à un changement très récent dans les dispositifs de formation sur lesquels interviennent ces formateurs, notamment au sein de la branche de la Propreté et Services Associés. En effet, cette dernière a récemment mis en place un socle commun. Par conséquent, elle a informé le 10 mai 2011 l'ensemble des OF habilités que le dispositif allait changer profondément en passant de formations appelées «Écrits professionnels » très axées sur l'individualisation à des formations dénommées $\mathrm{MCCP}^{14}$ directement liées au Référentiel des compétences clés en situation professionnelle (désormais RCCSP) et exclusivement orientées sur le travail et avec une finalité certifiante/diplômante. En fonction de ce changement, nous avons réalisé un questionnaire intégrant différentes thématiques pouvant éclairer notre recherche, notamment la connaissance et l'utilisation de référentiels linguistiques et autres (notamment RCCSP), les réactions relatives au socle commun, le changement ou non des pratiques de formation ${ }^{15}$.

14. Voir tableau 1.

15. Voir questionnaire en annexe. 


\section{Quelques résultats concernant l'adaptation des formateurs au nouveau contexte}

L'analyse des données recueillies a été traitée de manière qualitative. Peu de formateurs/coordonnateurs ont répondu (six réponses) et nous cherchions à prendre en compte effectivement les récits des formateurs face à ces changements. Cela nous a permis de les confronter aux données recueillies dans le journal de terrain. Les résultats analysés sont donc pris thématiquement en croisant régulièrement l'ensemble des données, ce qui devrait nous donner des pistes sur l'adaptation ou non des formateurs, d'autant plus qu'il est possible que les pratiques aient déjà évolué depuis la mise en place du RCCSP par l'ANLCI en mars 2009.

\section{Un référentiel à utiliser obligatoirement...}

L'outil étant en cours de développement au sein des OPCA, notamment depuis les opérations programmées par le FPSPP pour « favoriser l'acquisition d'un socle de connaissances et de compétences pour les salariés ${ }^{16} »$, les formateurs déclarent être dans l'obligation d'utiliser ce référentiel : « je suis dans un cadre dans lequel j'ai l'obligation de l'utiliser ${ }^{17}$ ». Nos résultats montrent que ce RCCSP est utilisé « à la demande des OPCA », certains conseillers préconisant de l'avoir « sur [sa] table de chevet $^{18}{ }^{»}$. Cependant nous ne sommes pas persuadé qu'il s'agisse d'un changement pour tous les formateurs, certains d'entre eux méconnaissant de nombreux référentiels (le CECR reste plus souvent cité mais il n'est pas pour autant utilisé sauf pour « indiquer le niveau linguistique / évaluer en fin de formation le niveau des stagiaires »). Ceci est à mettre en relation avec la formation initiale des formateurs : tous ne sont pas issus des sciences du langage, du FLE et de la didactique des langues et les résultats obtenus dans les questionnaires confirment ceux de l'étude menée par Frier (2009 : 82) sur les formateurs d'adultes :

16. Voir <http://www.fpspp.org/portail/portal/action/SimpleDownloadActionEvent/oid/00q-00001k-072> et <http://www.fpspp.org/portail/portal/action/ SimpleDownloadActionEvent/oid/00q-00001k-073> [en ligne, Réf. du 5 juin 2011] sur le site du FPSPP.

17. Sauf indication contraire, toutes les données présentées par la suite ont été recueillies dans le questionnaire.

18. Donnée recueillie dans le journal de terrain lors d'une réunion de présentation de la Maitrise des compétences clés de la Propreté le $1^{\mathrm{er}}$ avril 2011. 
le master FLE est très fortement représenté (45\%), suivi de près par les maitrises/DEA/masters dans des champs disciplinaires plus diversifiés : sciences de l'éducation, lettres modernes, info-com [...], l'un de nos témoins est ingénieur, un autre est titulaire d'une thèse en sciences.

De plus, leur entrée dans la vie active varie selon les différences générationnelles, certains partageant une histoire d'éducation permanente/éducation populaire et des référentiels savoirs de base $^{19}$, d'autres découvrant depuis peu ce type de formation.

Certains formateurs évoquent ainsi l'utilisation du RCCSP comme « une contrainte professionnelle » car ils ne peuvent pas faire sans, compte tenu des marchés de leurs OF avec les OPCA. Ce RCCSP est donc utilisé sans être réellement connu ( je ne le connais pas vraiment »), alors même que des formations à l'utilisation de ce référentiel existent au sein des branches, formations apparaissant souvent pour les formateurs comme une « sensibilisation à l'utilisation du RCCSP» alors que les dispositifs les présentent comme des « réunions d'information », des « journées de mutualisation » ou des « formations de formateurs $»^{20}$.

\section{S'adapter de toute façon}

Ainsi, même si son utilisation apparait contrainte, les formateurs déclarent en général qu'ils pourront « quand même travailler avec le référentiel », notamment parce qu' « il permet une meilleure mutualisation des supports / d'éviter d'oublier des objectifs à travailler / d'avoir une réflexion plus attentive à l'objectif visé et aux capacités qu'il mobilise / d'organiser, d'associer diverses compétences ». Ces réflexions de formateurs montrent qu'il existe, du moins pour certains d'entre eux, un début de changement des pratiques, notamment sur la mise en place d'une pédagogie sur objectifs en adéquation avec des objectifs de progrès professionnels (désormais OPP) fixés lors de l'analyse des besoins

19. On pense ici aux référentiels « Former les publics peu qualifiés : référentiel des savoirs de base, démarche référentielle de repérage des compétences » de Colette Dartois et Claude Thiry et au « Référentiel de formation linguistique de base » du CUEEP de Lille.

20. Respectivement pour la présentation du nouveau dispositif des Maitrise des compétences clés de la Propreté le $1^{\text {er }}$ avril 2011 pour le FAF Propreté / la réunion d'information et coordination Maitrise des savoirs fondamentaux du 6 février 2012 pour OPCALIM / la formation de formateurs du 3 février 2012 pour OPCALIA Propreté. 
des salariés et de la demande de l'entreprise. Cependant, cela s'avère assez compliqué pour eux : ils ont souvent le sentiment de ne pas avoir réellement assimilé cet outil perçu comme « trop dense ». La plus grosse critique faite à cet outil repose sur l'obligation de codifier les supports de formation en fonction du RCCSP, ceci dans le but de traiter l'ensemble des objectifs à atteindre. Cet avis est unanime; il peut être tranché : « on ne peut pas tout catégoriser, certaines choses se recoupent ou ne peuvent pas vraiment être catégorisées », dubitatif : « je ne comprends toujours pas très bien pourquoi certains items sont dans une rubrique et pas dans une autre / l'informatique est séparée de la communication écrite. Or on peut communiquer par écrit grâce à l'informatique ». Certaines réponses montrent que l'outil peut aussi poser problème dans son utilisation : «il n'est pas toujours aisé de rentrer dans une catégorie et une activité ne peut pas toujours être mise dans une case » et apparaitre à de nombreux égards comme une véritable « usine à gaz $»^{21}$. Il est vrai que lorsque l'on observe ce référentiel, on est frappé par sa composition dans la mesure où il mixe des activités à classer selon leur degré de complexité et leur niveau. On y trouve trois pôles de compétences (réalisation, interaction, initiative) déclinés en sept activités : exécuter une tâche, comprendre et justifier, communiquer, participer à une décision, faire face à un aléa, apprendre/tirer parti de l'expérience et faire preuve d'initiative. Cet ensemble varie selon trois degrés de capacités professionnelles : imitation, adaptation, transposition. Enfin le tout est découpé en quatre savoirs généraux (oral, écrit, calcul, espace/temps) et six savoirs appliqués (informatique (3 degrés), technologie (savoirs techniques), attitudes et comportements (trois degrés), gestes - postures - observation, réglementaire (sécurité, qualité) et ouverture culturelle). Le RCCSP totalise donc 154 cases à renseigner par des exemples d'objectifs à atteindre!

Cet outil complexe a été réalisé, selon ces auteurs « en partant des situations de travail bien réelles, nous avons analysé les tâches et formalisé les compétences de base exigées pour les remplir ${ }^{22} \gg$. Nous pouvons voir ici que la complexité de cet outil, qui répondrait donc à celle de l'activité professionnelle des salariés, a des limites. Les freins à l'utili-

21. Donnée recueillie dans le journal de terrain lors d'une formation de formateurs entre membres d'un réseau d'OF le 6 octobre 2011.

22. Extrait de la préface de la directrice de l'ANLCI, M.-T. Geffroy, du Guide d'utilisation du Référentiel des compétences clés en situation professionnelle version 1, Lyon, ANLCI, 2009. 


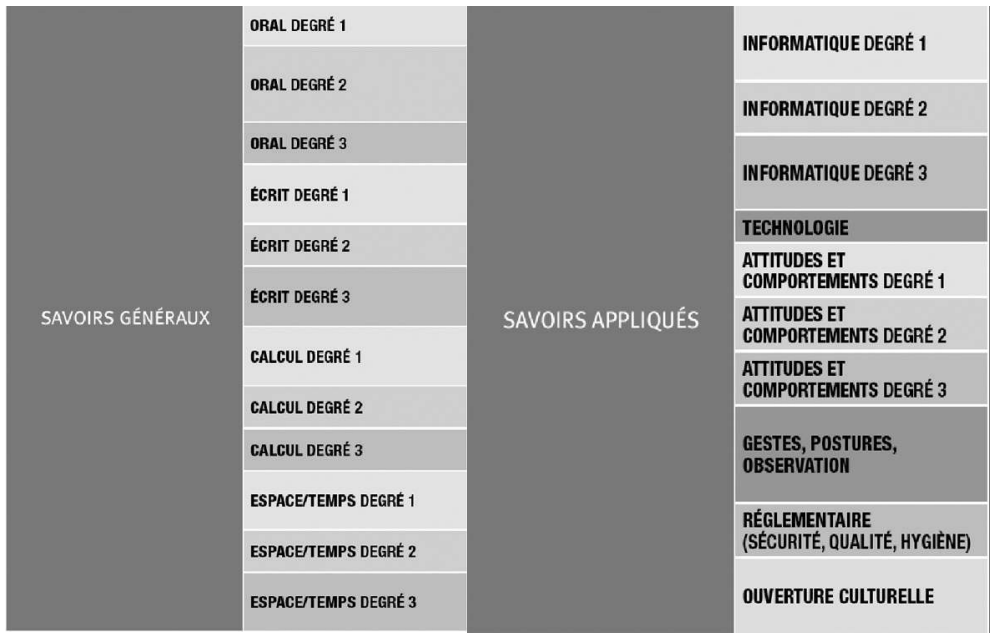

Tableau 2. - Les savoirs du RCCSP (ANLCI, 2009)

sation de cet outil peuvent avoir différentes origines et les résistances au changement ne sont pas étonnantes lors de toute modification organisationnelle dans un habitus professionnel. Nous sommes ici dans le cadre d'un changement planifié tel qu'il est défini par Collerette, Delisle et Perron (1997 : 94) dans lequel peut se déployer « l'expression implicite ou explicite de réactions de défense à l'endroit de l'intention de changement ». Or, ce changement prend ici une forme de nouveauté, dans le cadre d'une transition relativement rapide vers une autre conception de la formation pour les formateurs. Cependant, dans toute organisation, la temporalité est une constante inévitable, notamment quand ce changement peut sembler rapide. Nous pouvons nous demander si les formateurs des OF disposent du temps nécessaire à l'appropriation de cet outil et à sa déclinaison inévitable pour traduire des OPP en objectifs pédagogiques selon chaque branche, chaque contexte, chaque entreprise, chaque site et les personnes en formation. Cela suppose un temps important d'ingénierie en amont avec une analyse fine du travail de chaque salarié à former, une "évaluation des compétences langagières contextualisées » comme le précise Adami (2006 : 45). L'expérience de création d'un référentiel des compétences langagières pour les métiers de la propreté par l'équipe du CRAPEL/ATILF ${ }^{23}$ témoigne de ce travail énorme de recueil de données :

23. CRAPEL pour Centre de recherches et d'applications pédagogiques en langues / ATILF pour Analyse et traitement informatique de la langue française. 
entretiens avec des professionnels [...] visites de chantiers [...] enregistrements d'interactions sur le lieu de travail [...] [recueil] des documents écrits authentiques (fiches de poste, cahiers de liaison, fiches techniques, etc.) [...] photos des sites et notamment de la signalétique dans les bâtiments et sur divers panneaux d'affichage et d'information. (Adami, $2006: 45$ )

Il semblerait donc que cette ingénierie énorme relève de structures plus lourdes (comme des laboratoires de recherche par exemple) que des $\mathrm{OF}$. Une attention nouvelle devra être également portée à des formateurs majoritairement originaires du champ linguistique devant enseigner dans des domaines/savoirs comme la technologie ou l'informatique par exemple. Comme le précise Mangiante (2011 : 119), « l'ampleur de la tâche du formateur semble démesurée » et les formateurs en ont pleinement conscience car ils craignent de ne pas « appliquer correctement » le RCCSP. Et l'on perçoit ici une dérive consécutive à l'utilisation d'un tel outil : les formateurs vont-ils créer des supports pédagogiques adaptés aux salariés d'une entreprise avec toute sa particularité, son environnement contextuel ou vont-ils être amenés à concevoir des supports pour « coller aux référentiels / faire mousser le référentiel ${ }^{24}$ » ? Comme le dit un responsable pédagogique, le risque est une «vraie dérive du référen$\operatorname{tiel}^{25} \gg$ risquant de faire passer cet outil au service des formateurs, des entreprises et des personnes en formation à un outil formatant les dispositifs de formation. Ces formations pourraient ainsi s'inscrire dans une conception relativement différente de ce qu'elles étaient auparavant. C'est ce qu'il est possible d'observer avec la mise en place des socles communs.

\section{Vers des socles communs}

Les objectifs du FPSPP étant notamment de «favoriser l'acquisition d'un socle de connaissances et de compétences pour les salariés ${ }^{26} »$, différents OPCA ont répondu à cet appel à projet dit « socle », notamment tous les OPCA concernés par cette enquête. Dans leurs réponses à cet appel à projet, ils se sont engagés selon les termes suivants :

24. Donnée recueillie dans le journal de terrain lors d'une formation de formateurs entre membres d'un réseau d'OF le 6 octobre 2011.

25. Ibid.

26. Ces objectifs du FPSPP sont définis dans la loi 2009-1437 du 24 novembre 2009. 


\begin{tabular}{|l|l|}
\hline OPCA & ENGAGEMENT VIS À VIS DU SOCLE \\
\hline OPCALIM & $\begin{array}{l}\text { «Le socle commun de connaissances et de com- } \\
\text { pétences des industries agro-alimentaires (MSF } \\
\text { ALICIA) sera élaboré en réalisant un croisement du } \\
\text { référentiel RCCSP et des fiches métiers correspondant } \\
\text { aux certificats de qualification professionnelles. » }\end{array}$ \\
\hline OPCALIA & $\begin{array}{l}\text { «Ce projet innovant ayant pour objectif de définir, } \\
\text { valider puis déployer dans le secteur de la propreté et } \\
\text { des services associés un socle de compétence. » }\end{array}$ \\
\hline CONSTRUCTYS & $\begin{array}{l}\text { «Les formations prévues peuvent relever soit : } \\
\text { - des savoirs généraux (comprendre et se faire com- } \\
\text { prendre à l'oral, lire et écrire, calculer)...; } \\
\text { - des savoirs appliqués (se protéger, se situer dans } \\
\text { le temps et l'espace, comprendre l'environnement, } \\
\text { capacité à observer, prise en compte du développe- } \\
\text { ment durable...). » }\end{array}$ \\
\hline OPCALIA \\
FORTHAC & $\begin{array}{l}\text { «L'opération s'adresse en particulier aux salariés ne } \\
\text { maitrisant pas les compétences clés pour mobiliser } \\
\text { pleinement leurs capacités professionnelles. » }\end{array}$ \\
\hline
\end{tabular}

Tableau 3. - OPCA, RCCSP et Socle

Les formateurs intervenant dans ce cadre utilisent désormais le RCCSP et, de plus, ils vont devoir développer une didactique relativement à un socle commun transversal à des branches professionnelles, ce qui, selon un formateur, «permettra à tous les formateurs d'avoir les mêmes bases sur lesquelles travailler ». Auparavant, « on demandait à l'entreprise de renseigner les objectifs de progrès professionnels avec des progressions très diverses avec des objectifs plus ou moins définis, maintenant on aura des objectifs transversaux avec des spécificités si nécessaire ${ }^{27} \gg$. Ce type d'assertion fait douter certains formateurs voyant dans ces socles communs « une pratique idéale d'un métier et non pas la pratique concrète, dans un contexte précis ». Beaucoup d'entre eux s'interrogent alors sur la pertinence d'un tel outil : «pourquoi les entreprises et les personnes en formation adhéreraient-elles à cette approche ? » et sur sa finalité qui pourrait « devenir un moyen de pression de l'employeur face au salarié » car ces socles donneront lieu à certification.

27. Donnée recueillie dans le journal de terrain lors d'une réunion de présentation de la Maitrise des compétences clés de la Propreté le $1^{\mathrm{er}}$ avril 2011. 


\section{Conclusion : de nouvelles pratiques pour de nouvelles formations?}

Face à ces nouveaux enjeux, nous voyons que les formateurs s'interrogent beaucoup sur l'utilisation de tels outils et les répercussions que cela entraine dans les formations qu'ils mettent en place. Le processus étant en cours, il est difficile de savoir aujourd'hui si les pratiques effectives de formation changent. Cependant, notre enquête montre que les formateurs peuvent s'adapter, même si cette adaptation ne relève pas uniquement de leurs choix personnels. D'une part, leur degré de résistance au changement varie et par conséquence, ils ne s'inscriront pas tous de la même façon dans ce mouvement. D'autre part, il apparait un changement réel du métier de formateur en situation professionnelle qui ne fait plus tout à fait la même chose. Alors qu'auparavant, les formateurs définissaient des objectifs pédagogiques individualisés selon les besoins des salariés et la demande d'une entreprise, dorénavant ils devront former un groupe avec des objectifs communs dans la perspective que tous obtiennent une certification de branche. Cette différence de perspective pourra entrainer des difficultés pour que les formateurs adaptent leurs pratiques dans des conditions profitables à tous $(\mathrm{OF}$, formateurs, entreprises, salariés et OPCA). Si les formateurs ne sont pas suffisamment accompagnés, certaines pratiques de formation pourraient devenir très instrumentalisées, faisant du RCCSP un outil niant complètement les stagiaires en formation et faisant de ce référentiel de compétences un super référentiel à tout faire : professionnel / de compétences / de formation / d'évaluation et de certification, un « monument technique au détriment de la souplesse » comme le souligne Aubret (2009 :181). Nous doutons que tous les formateurs participent à ces changements, y adhèrent, y trouvent leur compte au risque de voir une communauté de pratiques (dans le sens où le conçoit Wenger, 2000) se déliter. C'est pour cela que nous pensons nécessaire l'accompagnement au changement, notamment en privilégiant la mise en place de moments collaboratifs où chacun pourrait développer la réflexivité nécessaire à cette évolution en cours des pratiques. Pour cela, nous pensons que les démarches portfolio développées par Muriel Molinié (2011) pourraient constituer un accompagnement utile tout au long de ce cheminement des formateurs en pleine mobilité professionnelle. Ces derniers pourraient ainsi, à travers un processus constructiviste, développer une méthodologie favorisant leur implication. Afin d'éviter la situation de déséquilibre ressentie par un formateur se décrivant comme « un acrobate qui avance sur un 
fil », ce type de démarche de « seconde génération » de portfolio, comme le précise Molinié (2011 : 56), permettrait de « devenir un puissant dispositif de formation et un vecteur de développement d'expériences et de compétences » au service des formateurs et, par ce biais, des dispositifs de formation au sein desquels ils officient.

\section{RÉFÉRENCES BIBLIOGRAPHIQUES}

AdAmi H. (2006) : « Construire un référentiel des compétences langagières pour les métiers de la propreté », Rencontres DGLFLF Apprendre le français dans un contexte professionnel, Séminaire de réflexion 2-3 juin 2006, p. 43-49.

AdAmi H. (2009) : La formation linguistique des migrants, Paris, CLE International.

Adami H. (2010) : «Introduction », dans H. Adami, S. Etienne et A. Bretegnier (coord.), Littéracie et alphabétisation des adultes : questions théoriques, andragogiques et didactiques, Savoirs et Formation - Recherches \& Pratiques, $\mathrm{n}^{\circ} 1$, Montreuil, AEFTI.

Aubret J. (2009) : «Compétences, formations et métiers du FLE », Cahiers de l'ASDIFLE, n 20, p. 174-183.

ANLCI (2009) : Référentiel des compétences clés en situation professionnelle version 1, Lyon, ANLCI.

ANLCI (2009) : Guide d'utilisation du Référentiel des compétences clés en situation professionnelle version 1, Lyon, ANLCI.

Boutet J. (2006) : «Usages professionnels de la parole : le cas des centres d'appel », Rencontres DGLFLF Apprendre le français dans un contexte professionnel, Séminaire de réflexion 2-3 juin 2006, p. 38-42.

BRETEGNIER A. (2011) : Formation linguistique en contexte d'insertion. Compétences, posture, professionnalité : concevoir un cadre de référence(s), Berne, Peter Lang.

Bretegnier A., Etienne S. et Adami H. (2011) : « Définir le champ de la formation linguistique en contextes d'insertion », dans A. Bretegnier (dir.) : Formation linguistique en contexte d'insertion. Compétences, posture, professionnalité : concevoir un cadre de référence(s), Berne, Peter Lang.

CAmbra Gine M. (2003) : Une approche ethnographique de la classe de langue, Paris, Didier.

Collerette P., Delisle G. et Perron R. (1997) : Le changement organisationnel : théorie et pratique, Sainte-Foy, Presses de l'université du Québec. CUEPP (1996) : Référentiel de formation linguistique de base du CUEEP, Lille, FAS. 
Dartois C. et Thiry C. (2000) : Former les publics peu qualifiés : référentiel des savoirs de base, démarche référentielle de repérage des compétences, Paris, La Documentation française.

FrIER C. (2010) : «Être formateur d'adultes aujourd'hui : un équilibre fragile entre idéal et processus de construction de l'identité professionnelle », dans H. Adami, S. Etienne et A. Bretegnier (coord.), Littéracie et alphabétisation des adultes : questions théoriques, andragogiques et didactiques, Savoirs et Formation - Recherches \& Pratiques, n 1 , Montreuil, AEFTI, p. 79-92.

Géraud M.-O., Leservoisier O. et Pottier R. (1998) : Les notions clés de l'ethnologie, Paris, Armand Colin.

Goffman E. (1961) : Asylums, New-York, Anchor Books.

Gumperz J. et Hymes D. (1964) : The Ethnography of Communication, American Anthropologist, Special Publication, Washington DC, American Anthropological Association.

Hymes D. (1974) : Foundations in Sociolinguistics. An Ethnographic Approach, Philadelphia, University of Pennsylvania Press.

Malinowki B. (1963) : Les Argonautes du Pacifique occidental, Paris, Gallimard.

Mangiante J.-M. (2011) : «Élaborer une analyse des besoins », dans A. Bretegnier (dir.), Formation linguistique en contexte d'insertion. Compétences, posture, professionnalité : concevoir un cadre de référence(s), Berne, Peter Lang.

Maroun E. (2006) : «La professionnalisation des intervenants », Rencontres DGLFLF Apprendre le français dans un contexte professionnel, Séminaire de réflexion 2-3 juin 2006, p. 72-74.

Molinie M. (2011) : «Construire la relation interculturelle en mobilité internationale : entre formation académique et enjeux de professionnalisation », dans Démarches portfolio en didactique des langues et des cultures Enjeux de formation par la recherche action, Cergy-Pontoise, CRTF.

Mourlon-Dallies F. (2008) : Enseigner une langue à des fins professionnelles, Paris, Didier.

WENGER E. (2000) : Communities of practice: learning, meaning and identity, New York, Cambridge University Press.

Winkin Y. (2001) : Anthropologie de la communication. De la théorie au terrain, Paris, De Boeck Université/Éditions du Seuil.

\section{ANNEXES}

I. - Enquête qualitative formateur

II. - Tableau des corpus recueillis (extraits) 
Annexe I. - Enquête qualitative formateur

DANS LE CADRE D'UNE RECHERCHE UNIVERSITAIRE EN COURS, NOUS VOUS INVITONS À RÉPONDRE AUX QUESTIONS SUIVANTES. NOUS VOUS REMERCIONS POUR VOTRE AIDE.

Vous pouvez me le renvoyer sur adresse courriel

\section{Généralités}

Pouvez-vous renseigner vos coordonnées s'il vous plait? Ces données resteront confidentielles. Elles me servent uniquement pour me donner une image du profil des formateurs/formatrices et pour vous contacter en cas de besoin.

Nom :

Prénom :

Âge :

Sexe :

Entreprise/Structure :

Poste occupé actuellement :

Adresse de courriel :

\section{Formations et expériences professionnelles}

Quel est votre cursus initial?

Types et noms d'établissement (écoles, universités, institutions...)

Quels en étaient les principaux contenus? \begin{tabular}{l|l} 
Diplômes, certificats, & Disciplines
\end{tabular}

Cette ou ces formation(s) sont-elles en adéquation avec votre parcours professionnel?

Pensez-vous que votre formation initiale vous a suffisamment préparé pour occuper votre poste actuel? Expliquez pourquoi.

Quels emplois avez-vous occupés avant votre poste actuel?

Depuis quand travaillez-vous au sein de votre structure?

Avez-vous changé de poste dans votre structure? Si oui, détaillez votre parcours.

Avez-vous suivi des formations, dans le cadre de la formation professionnelle continue, pour vous perfectionner, acquérir de nouvelles compétences, etc. ? Précisez.

\begin{tabular}{l|l|l}
$\begin{array}{l}\text { Type et nom d'établissement } \\
\text { (écoles, universités, institutions, } \\
\text { Récif, OPCA, CIEP...) }\end{array}$ & $\begin{array}{l}\text { Diplômes, certificats, } \\
\text { qualifications, } \\
\text { habilitations obtenus }\end{array}$ & Disciplines, thèmes \\
\hline
\end{tabular}

Ces formations ont-elles été bénéfiques ou pas? Expliquez pourquoi.

\section{Publics}

Quels publics (âge, sexe, projets...) formez-vous dans votre structure? 
Comment catégorisez-vous au niveau linguistique les publics que vous formez dans votre structure? Détaillez.

Pensez-vous que ces catégories linguistiques soient pertinentes? En quoi?

Avec quels OPCA et entreprises travaillez-vous?

Quels publics (âge, sexe, projets...) formez-vous personnellement dans votre structure?

Relativement aux personnes que vous recevez en formation, travaillez-vous différemment? (Expliquez : changement de méthodes, d'outils d'ingénierie de formation et d'ingénierie pédagogique, etc.)

Faites-vous une différence entre salariés et demandeurs d'emploi ? Expliquez.

Avez-vous ressenti l'effet de la loi du 4 mai 2004 (le français est devenu compétence professionnelle, inscription dans le code du travail)? Si oui, qu'est-ce qui a changé au sein de votre structure?

Si oui, qu'est-ce qui a changé pour vous?

Pensez-vous qu'il faille posséder des savoirs et savoir-faire complémentaires, différents... pour accueillir les salariés?

Comment participez-vous à la mise en place des formations dans vos structures? (quelles sont vos missions exactes?)

\section{Modalités pédagogiques}

Quels sont les contenus que vous enseignez aujourd'hui?

Ces contenus ont-ils évolué ou non au fil des ans? Pourquoi «oui » et/ou pourquoi «non»?

Si vous avez adapté les contenus en fonction des publics reçus, essayez d'expliquer le cheminement personnel et au sein de l'équipe qui s'est mise en place.

Cette évolution de vos pratiques vous convient-elle? Expliquez.

\section{Référentiels en général}

Quels référentiels connaissez-vous?

Avez-vous participé et/ou participez-vous à l'élaboration de ces référentiels? Si oui, pourquoi et comment?

Quels référentiels utilisez-vous? Pourquoi?

Créez-vous vos ressources en fonction de ces référentiels? si oui, quelles ressources et pourquoi? 


\section{Le Référentiel des compétences clés en situation professionnelle (RCCSP)}

Utilisez-vous ce référentiel plus que d'autres? Pourquoi? Avec quels OPCA ? Quels publics?

Avez-vous été formé à l'utilisation de ce RCCSP? Si oui, en quoi cette formation a-t-elle été pertinente ou non?

En quoi l'utilisation de ce RCCSP a-t-elle changé vos pratiques de formation?

Que pensez-vous de ce RCCSP ? Soyez le plus clair et le plus franc possible.

Que signifie, pour vous, « socle commun de connaissances et de compétences »? Qu'en pensez-vous?

Plusieurs OPCA constituent de tels socles. Qu'en pensez-vous?

Pensez-vous que ce type de socle va changer vos façons de travailler? Expliquez.

Pour celles et ceux qui interviennent sur des actions du FAF Propreté. Avez-vous été formé à la "Maitrise des compétences clés de la Propreté" ? Que pensez-vous de cette formation?

Pour celles et ceux qui interviennent sur des actions du FAF Propreté. En quoi le passage de la formation «Écrits professionnels » à «Maitrise des compétences clés de la Propreté » va-t-il changer les choses pour vous?

Pour tout le monde, comment percevez-vous aujourd'hui votre métier de formateur?

Pour tout le monde, avez-vous envie de changer de métier? Pourquoi?

Merci beaucoup à toutes et tous 


\begin{tabular}{|c|c|c|c|c|c|}
\hline 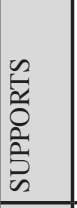 & 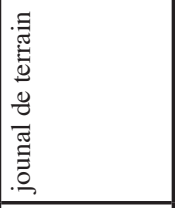 & 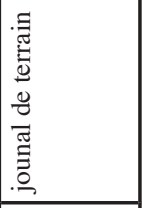 & 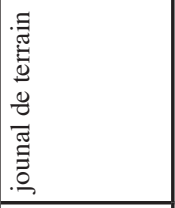 & 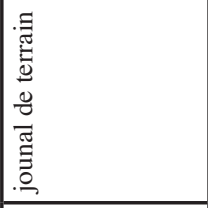 & 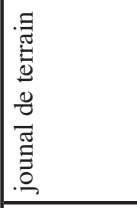 \\
\hline 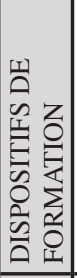 & 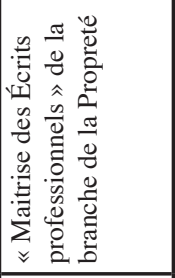 & 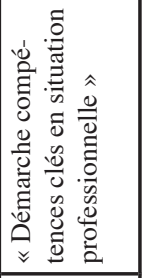 & 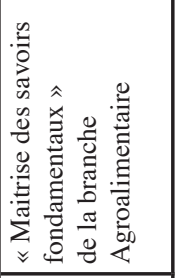 & 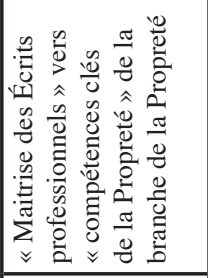 & 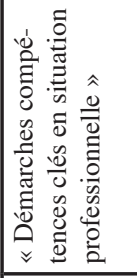 \\
\hline 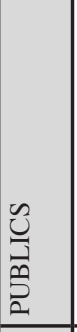 & 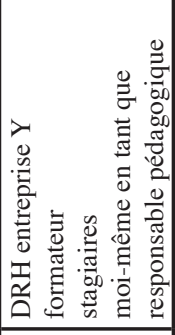 & 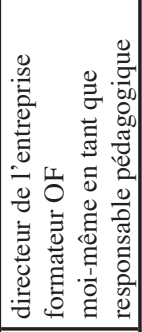 & 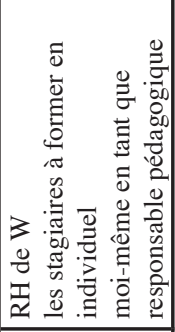 & 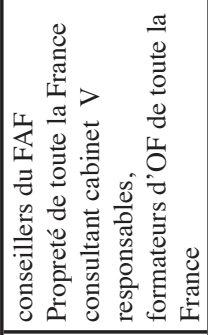 & 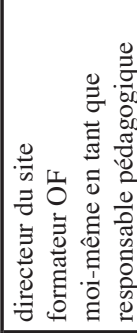 \\
\hline 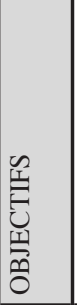 & 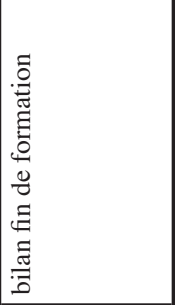 & 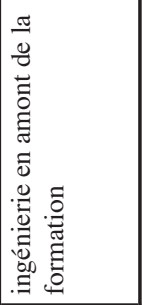 & 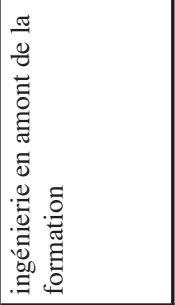 & 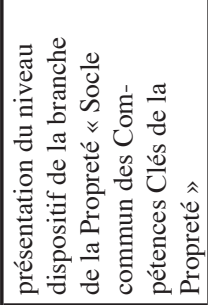 & 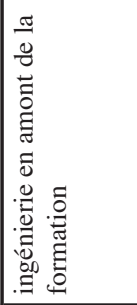 \\
\hline 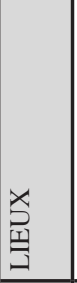 & 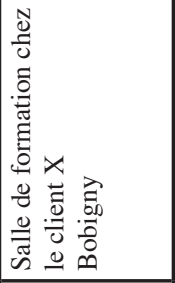 & 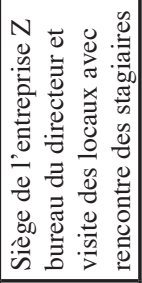 & 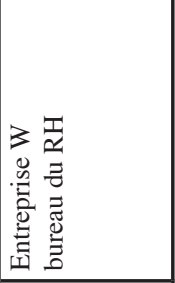 & 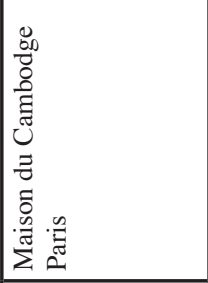 & 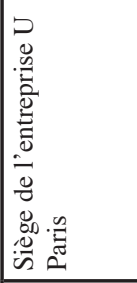 \\
\hline 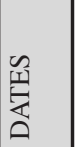 & 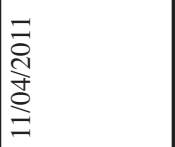 & 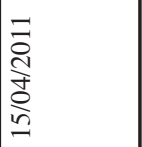 & 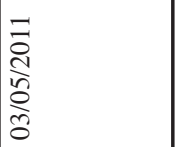 & 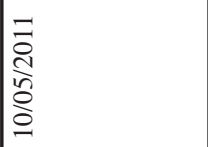 & 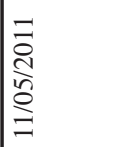 \\
\hline
\end{tabular}




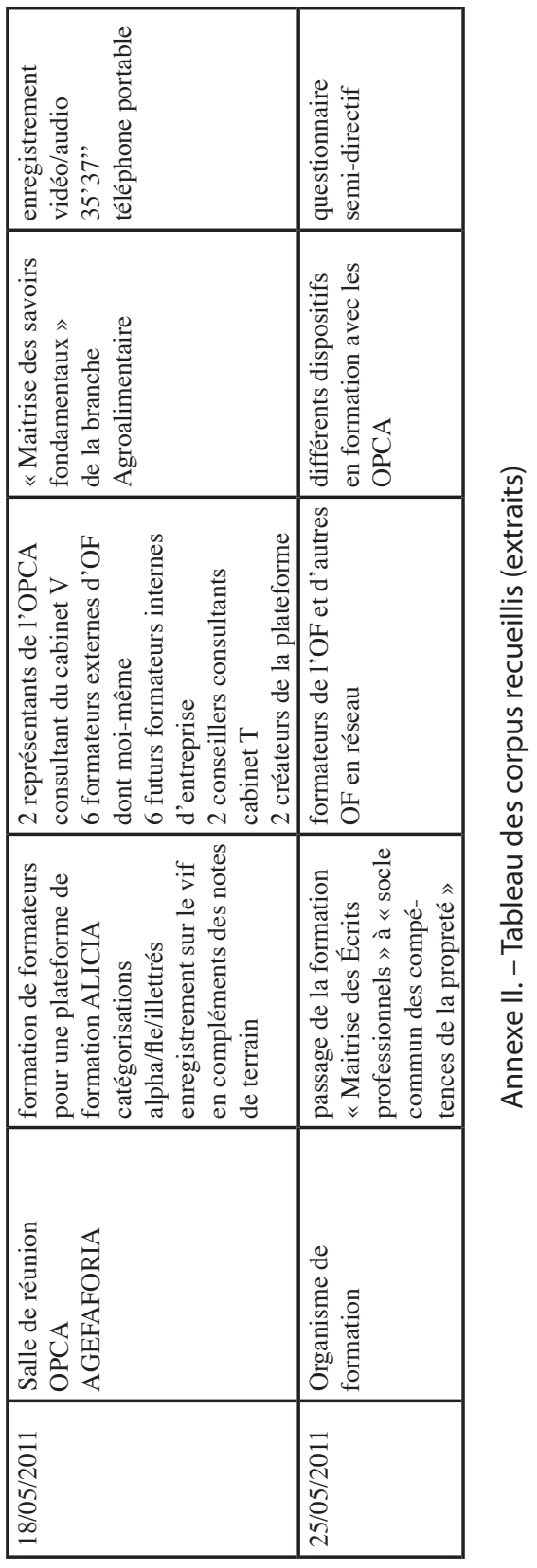


